\title{
Assessment and Early Identification of Young Children with Social Emotional Difficulties and Behavioral Challenges
}

\author{
Elizabeth A. Steed ${ }^{1, *}$ and Rashida Banerjee ${ }^{2}$ \\ ${ }^{1}$ University of Colorado Denver, USA \\ ${ }^{2}$ University of Northern Colorado, USA
}

\begin{abstract}
This paper discusses the issues and challenges to the assessment and early identification of social emotional and behavioral issues in young children birth through five years of age in the United States. The linked system framework of social emotional assessment and intervention [1] is outlined, and recommendations for authentic assessment of social emotional development are provided to assist professionals in identifying young children early for amelioration and prevention of behavioral challenges.
\end{abstract}

Keywords: Social emotional, challenging behavior, screening, assessment, early intervention, linked system.

Very young children with social emotional difficulties and behavioral challenges have received increased attention from researchers, government agencies, and educational leaders in the United States [2]. Unaddressed early social emotional issues are associated with long-term negative consequences such as problematic relationships with parents, peers, and teachers and academic failure [3]. Approximately $5 \%$ of young children birth through five years of age may experience "extreme functional impairment" due to an emotional or behavioral disorder [4], and approximately 10 to $15 \%$ of young children have mild to moderate social emotional difficulties or challenging behavior [5]. These prevalence rates increase to 20 to $33 \%$ when young children live in poverty [6] and up to almost $50 \%$ for young children with completed child welfare investigations for abuse or neglect [7].

Given these prevalence rates, early identification and intervention is imperative to effectively and efficiently address social emotional difficulties and behavioral challenges when children are very young. Currently, identification of young children with social emotional issues lags behind identification of young children with developmental delays in cognitive, motor, or language domains [8]. This paper will highlight some common obstacles to effective assessment and early identification of social emotional issues, share a framework for linking social emotional assessment to intervention, and provide recommendations for using authentic assessment to identify and support young children with social emotional difficulties and behavioral challenges and their families.

*Address correspondence to this author at the University of Colorado Denver, USA; E-mail: Elizabeth.steed@gmail.com

\section{OBSTACLES TO EFFECTIVE ASSESSMENT AND EARLY IDENTIFICATION}

There are several obstacles to effective assessment and early identification of social emotional difficulties, including inadequate professional training, lack of understanding of cultural variations in social emotional development, limited social emotional screening tools for young children, and a dearth of community-based social emotional services for young children.

\section{Inadequate Training in Social Emotional Issues}

First, there are two main pathways to screening and early identification of social emotional concerns in young children in the United States: (a) health care, and (b) early care and education [9]. In the first pathway, medical professionals, including pediatricians, who interact with young children birth through five and their families are in a prime position to support families to identify when children's social emotional issues are in need of further evaluation [10]. Pediatricians are responsible for providing scheduled surveillance and screening for all developmental issues, including social emotional concerns and providing referrals for social emotional or mental health services as necessary [11]. Unfortunately, pediatricians and other medical personnel often fail to identify when young children have a serious social emotional issue [12]. Research has indicated that pediatricians identify half, or less than half, of children with social emotional issues and behavioral disorders [13]. The referral rate to mental health services is especially low for infants and toddlers [11].

Pediatricians and other primary care physicians face various challenges to identifying social emotional difficulties, including limited staff and time to administer 
social emotional screening instruments, a lack of training in interpreting the scores, and the perception of a lack of referral agencies to send the families to if there is a positive screen [14, 15]. Further, young children may not demonstrate the social emotional issues that their families see in other settings in their doctor's office during a short visit. Additionally, families may be reluctant to bring up social emotional concerns, focusing instead on their young children's physical health when communicating with the physician [16].

The second pathway to screening and early identification of early care and education includes various professionals who interact with young children birth through five, such as childcare directors and teachers. These professionals are not well trained to identify social emotional issues early $[9,17]$. There are federally funded programs (e.g. Head Start) that require regular social emotional screenings for young children; however, most children in early care environments are not in programs that provide these services [9]. In other pathways for screening and early identification, such as child welfare services, there are few mandates for regular social emotional screening and surveillance [9]. In sum, children with serious social emotional concerns may not be identified as early as they could have been, thereby missing out on services to address their behavioral issues [18].

\section{Cultural Variations in Social Emotional Development and Behavioral Expectations}

In addition to professionals not being trained in young children's social emotional issues, there are cultural variations in social emotional development and behavioral expectations that complicate the task of deciphering whether or not a social emotional issue is a serious concern. When and how to display emotions, how to cope with stress or change, and when it's appropriate to seek adult attention are all examples of social behaviors that children learn through their culture [19]. For young children, these social behaviors are learned through their early relationships with their primary caregivers [20].

Cultural differences between families' and professionals' social and behavioral expectations may lead to misunderstandings [19]. For example, a child whose family has taught her to respect others by being quiet and avoiding eye contact around adults may be misunderstood by professionals as unresponsive and lacking social skills. Cultural misunderstandings may negatively impact collaborative relationships with families and/or children may be misidentified as having a serious social emotional or behavioral issue when they do not or vice versa. There may be additional issues around identification and intervention for social emotional challenges due to different beliefs across race and ethnicities regarding the causes of social emotional difficulties [21]. A lack of cultural diversity amongst health, mental health, and the early childhood fields presents a barrier to communication, engagement, and trust between families and professionals during the assessment and early identification process [21].

\section{Lack of Social Emotional Screening Tools}

Another challenge is the lack of social emotional screening tools developed for very young children that are reliable, valid, and usable. The AAP Task Force on Mental Health [22] recommends using validated screening assessments for universal screening of social emotional difficulties during all young children's well-child visits. Compared to two decades ago, there are now more available specialized social emotional screening tools such as the Ages and Stages Questionnaires: Social-Emotional (ASQ:SE) [23], the Brief Infant-Toddler Social Emotional Assessment (BITSEA) [24], and the Temperament and Atypical Behavior Scales Screener (TABS) [25] to identify social emotional difficulties in very young children. However, the social emotional screening tools that are available for use with infants, toddlers, and preschoolers often fall short in either technical adequacy or in usability [26], or are expensive and logistically difficult to administer [27].

The lack of cost effective, quick, and usable screening tools may explain the phenomenon that many social emotional early identification approaches for children birth through five are not systematic, and do not involve formal screening procedures [28]. There are some positive aspects of currently available screening tools. First, they use input from families and provide specific forms to facilitate the assessment of young children's social emotional behaviors across settings, such as childcare centers and home environments per the Division for Early Childhood's Recommended Practices [29]. They have relatively culturally diverse samples. And, finally, they provide specific recommendations (e.g., modified or eliminated questions) for assessing children and families from diverse cultural and linguistic backgrounds [30]. 


\section{Lack of Community-Based Social Emotional Services for Young Children}

Even when universal social emotional screening instruments are used, there is a paucity of communitybased social emotional or mental health services for young children once a child is identified as needing such services [1]. Many professionals report low rates of using universal screening for social emotional issues due to a lack of or a perceived lack of places to refer children if there is a positive screen [31]. Approximately 2 to $3 \%$ of young children use social emotional or mental health services; this is a fraction of the children that are known to need such services [32]. Missing out on needed social emotional or mental health services disproportionally affects young children of color and children whose families do not carry insurance [32]. The majority of children who do receive social emotional or mental health services receive them in school [33]. So, for young children birth through five who are not yet part of the public school system, they are at the mercy of fragmented, disjointed, or absent partnerships between medical professionals, early childhood systems of service delivery, and communitybased mental health providers.

\section{LINKED SYSTEM OF SOCIAL EMOTIONAL ASSESSMENT}

In order to address the various systemic and logistical challenges to assessment and early identification of social and emotional difficulties in young children, integrated and efficient processes to screen and connect young children to social emotional or mental health services are necessary [27]. This linked system will need to include formal screening and assessment tools, collaboration between and across systems, and culturally responsive practices at each step to address the needs of children and families from diverse cultural and linguistic backgrounds.

\section{Components of the Linked System}

The linked system framework of early identification and intervention [34] for social emotional development has five processes that are interrelated: (1) Screening, (2) Assessment, (3) Goal Development, (4) Intervention, and (5) Evaluation. Squires and Bricker [1] refined the linked system to include processes related to social emotional screening, assessment, goal development, intervention, and evaluation that forms the basis for the Activity-Based Intervention: Social Emotional (ABI:SE) Approach.

In the two most often used pathways to screening and early identification in health care and early care and education, a child is most commonly referred for social emotional concerns by a pediatrician or a parent or caregiver [9]. Then, the pediatrician, family member, and/or caregiver complete a formal screening instrument. If the child has a positive screen and is determined to need a referral for social emotional services, a curriculum-based and authentic assessment is completed that is focused on social emotional development, such as the Social Emotional Assessment Measure (SEAM) [35]. The use of a specialized valid and reliable curriculum-based assessment allows for the next step in the linked system, the development of social emotional goals that caregivers (e.g., parents, guardians, childcare teachers) may implement in daily routines. Intervention based on the goals should occur throughout the day and across the activities children engage in with attention to the cultural relevance for children and families. For example, a caregiver may support a child's use of self-regulation strategies during diapering and feeding and thus, do so multiple times a day in meaningful, developmentally appropriate ways. Finally, the child's response to the intervention should be regularly measured with social emotional progress monitoring tools so that adjustments to social emotional goals and supports may be made [1].

\section{RECOMMENDATIONS}

There are a number of evidence-based and promising strategies that may assist professionals in each step of the linked system framework for social emotional screening, assessment, goal development, intervention, and evaluation. In describing these strategies, we particularly refer to the: (a) NAEYC and NAECS/SDE Joint Position Statement on Early Childhood Curriculum and Assessment [36], (b) Division for Early Childhood's (DEC) position statement on Identification of and Intervention with Challenging Behavior [37], and (c) Division for Early Childhood's Recommended Practices [29]. In the following three sections we describe strategies, that when combined, will assist professionals in the assessment and early identification of social emotional difficulties and behavioral challenges in young children, including those from culturally and linguistically diverse backgrounds.

\section{Use Multiple and Authentic Social Emotional Assessment Methods}

Using standardized tools for the assessment of young children's social emotional skill can be 
inappropriate, because young children respond inconsistently to unfamiliar settings, tasks, and providers [38]. This leads to the questionable reliability and validity of scores from standardized social emotional assessments that are administered by professionals unknown to the child, in clinical settings and with novel materials and activities. To address this issue, researchers have advocated for using multiple and authentic assessment methods to more accurately determine whether children have achieved key social emotional developmental milestones and/or have clinically significant social emotional difficulties or behavioral challenges [39].

Authentic social emotional assessments gather information from key family members and caregivers, involve observations within familiar settings (e.g., home, childcare classrooms) within typical routines (e.g., mealtime) or activities (e.g., play on the floor), and take into consideration children's experiences and background [18]. This authentic assessment approach is essential for obtaining a more accurate picture of young children's social emotional development and issues of concern.

Health care and early care and education professionals concerned about child's social emotional development may use specific authentic assessment methods such as: (a) observation of children during typical routines in a familiar environment with familiar adults and peers, (b) Ethnographic Interviewing [40] and Routines Based Interviewing and Assessment Strategies [41] that allow families to provide critical information about children's performance during routines and share their specific concerns and priorities, (c) review of past records such as medical records and educational reports that provide historical information about children's social emotional skills, and (d) and collection and review of data from formal social emotional assessments conducted by various multidisciplinary team members who have observed the child in a variety of settings over time [39]. Thus, multiple and authentic social emotional assessment methods are used in a linked system to then develop goals for the child that address their needs and family priorities.

\section{Choose Appropriate Social Emotional Assessment Tools}

It is important to use formalized processes to assess and identify young children with social emotional difficulties and behavioral challenges and to choose the appropriate tools that assess the various components of the linked system framework [36,38]. For example, before beginning any new assessment, the assessment team must clearly identify the function and purpose of the assessment (e.g. screening, determining eligibility for services, individualized planning, or monitoring child progress). The assessment tools and methods used (e.g. behavior rating scales, direct assessment, criterion-referenced, norm-referenced, naturalistic observations) must match the purpose. Furthermore, the assessment materials and strategies that are used must be reliable, valid, and normed on the culture and environment they will be used in. The tools must be sensitive, and "appropriate for the child's age and level of development and accommodate the child's sensory, physical, communication, cultural, linguistic, social and emotional characteristics" [29].

Informal assessments methods, such as semistructured interviews may be used to collect information regarding multiple factors including information about the families' routines and expectations and the children's behavior and performance across activities, people, and environments. You may see an example at http://csefel.vanderbilt.edu/modules/module3a/handout 5.pdf, and see Henderson and Strain [30] for details about the utility, acceptability, authenticity, equity, congruency, sensitivity, time, and cost of various screening and assessment tools for young children's social emotional development.

In addition to the formal and informal assessment tools, clinical judgement should be used to determine child's current performance and next steps in determining eligibility and intervention [29]. Informed opinion based on rich behavioral observations assists in gathering realistic and natural information from those who are familiar with the child; this strategy when combined with other sources of information will assist in screening for social emotional concerns in young children.

\section{Partner with Families to Address the Social Emotional Needs of their Child(ren)}

Given that behavior is culturally defined and situated within the family and community expectations, it is critical that families are effective and active partners when conducting social emotional screening and assessment [18, 42]. The importance of collaboration across all environments the child encounters, specifically the home, school, and 
community is critical [43]. IDEA recognizes this significance by including families as active members of a child's multidisciplinary team (IDEA C.F.R. 34 $\S 303.24)$. Professionals are often unfamiliar with the children prior to the screening and thus may not get the true picture of the child's skills and needs. Families know their children best and can best speak to children's different social emotional and behavioral issues across contexts. Further, family members may perform various roles during the assessment process to help the assessment team gather reliable and valid information to be able to make meaningful and accurate program planning decisions. Active family involvement may also aid in addressing challenges associated with understanding social emotional development in children from linguistically and culturally diverse backgrounds.

When working with families who are non-native English speakers, assessment teams need to develop strategies for working with interpreters and cultural mediators. A number of specific strategies for effectively working with interpreters have been described in the literature $[44,45]$. They include accessing well-trained and biliterate interpreters, developing a team approach with the interpreter, planning before sessions and debriefing afterward, and identifying the specific type of interpretation that will be utilized. Furthermore, in order to understand the cultural context of the children's behavior and family expectation, participation of a cultural mediator as a team member during assessment is recommended. A cultural mediator is knowledgeable about language and culture of both the family and the program and translates between the culture of the environment in which services are provided and the child's family in order to produce understanding, share information and create relationships between the family and the interventionists [42].

Finally, upon the completion of the assessment, the results need to be presented in a timely, sensitive, jargon free, and strengths based way that can be easily understood by the families [29,42]. Typically, one team member may be appointed to share the results with the family both verbally and in writing for families' future reference. Partnering with families to address their child's social emotional growth and development is used across all parts of a linked system. When developing functional goals for the Individual Family Service Plan or the Individualized Education Plan, team members, including family members, take into account family's priorities and child's interest and participation in daily activities. Once the goals are developed, the team members identify the needed supports to meet these goals that maximize family members' confidence and competence.

\section{CONCLUSION}

There is increased awareness of the social emotional needs of our youngest children. It is important that we develop and utilize linked systems of authentic assessment and evidence-based early intervention that will efficiently, effectively, and collaboratively direct young children and their families to needed services to ameliorate challenging behavior and develop key social emotional skills. This task - to expand and improve our screening, assessment, and intervention approach - is imperative given the high rates of young children experiencing social emotional difficulties and challenging behavior in their homes and childcare settings. More work needs to be done on how to integrate social emotional or mental health services into pediatrician and primary care practices that are often families' first point of contact for very young children with social emotional difficulties and behavioral challenges [11].

Based on research and experience in the field, we described a number of strategies that can support reliable and valid social emotional assessment, goal development, intervention, and evaluation in a seamless service delivery system for very young children. Use of these strategies may enhance current assessment practices and result in earlier identification and positive outcomes for children with social emotional needs. Assessment of the social emotional skills of young children is a multidisciplinary responsibility, involving early intervention/ early childhood special education (El/ECSE) professionals, health professionals, and related service providers such as mental health consultants, speech and language pathologists, and social workers. We need and must do better, especially for the children and families who need it most.

\section{REFERENCES}

[1] Squires J, Bricker D. An activity-based approach to developing young children's social emotional competence. Baltimore, MD: Paul Brookes Publishing 2007.

[2] McCabe L, Frede EC. Challenging behaviors and the role of preschool education: Preschool policy brief. Washington, DC: National Institute for Early Education Research 2007.

[3] Carter AS, Briggs-Gowan MJ, Davis NO. Assessment of young children's social-emotional development and psychopathology: Recent advances and recommendations. 
The Journal of Child Psychology and Psychiatry 2004; 45: 109-134.

http://dx.doi.org/10.1046/j.0021-9630.2003.00316.x

[4] National Institute of Mental Health. Report of the Surgeon General's conference on children's mental health: A national action agenda. Washington, DC: Department of Health and Human Services 2000.

[5] Campbell S. Behavior problems in preschool children: A review of recent research. Journal of Child Psychology and Psychiatry 1995; 36: 113-149.

http://dx.doi.org/10.1111/j.1469-7610.1995.tb01657.x

[6] Qi C, Kaiser A. Behavior problems of preschool children from low-income families: A review of the literature. Topics in Early Childhood Special Education 2003; 23: 188-216. http://dx.doi.org/10.1177/02711214030230040201

[7] Burns. et al. Mental health need and access to mental health services for youth involved with child welfare: A national survey. Journal of the American Academy of Child and Adolescent Psychiatry 2004; 43: 960-970.

http://dx.doi.org/10.1097/01.chi.0000127590.95585.65

[8] Horwitz SM, Gary LC, Briggs-Gowan MJ, Carter AS. Do needs drive services use in young children. Pediatrics 2003; 112: 1373-1378.

http://dx.doi.org/10.1542/peds.112.6.1373

[9] Powell D, Fixsen D, Dunlap G, Smith B, Fox L. A synthesis of knowledge relevant to pathways of service delivery for young children with or at risk of challenging behavior. Journal of Early Intervention 2007; 29: 81-106. http://dx.doi.org/10.1177/105381510702900201

[10] American Academy of Pediatrics, Committee on Psychosocial Aspects of Child and Family Health and Task Force on Mental Health. Policy statement - The future of pediatrics: Mental health competencies for pediatric primary care. Pediatrics 2009; 124: 410-421. http://dx.doi.org/10.1542/peds.2009-1061

[11] Godoy L, Carter AS, Silver RB, Dickstein S, Seifer R. Infants and toddlers left behind: Mental health screening and consultation in primary care. Journal of Developmental \& Behavioral Pediatrics 2014; 35: 334-343. http://dx.doi.org/10.1097/DBP.0000000000000060

[12] Jellinek M, Patel BP, Froehle MC. Bright futures in practice: Mental health. Volumes 1 and 2. National Center for Education in Maternal and Child Health. Washington, DC: Georgetown University 2002.

[13] Perrin E, Stancin T. A continuing dilemma: Whether and how to screen for concerns about children's behavior. Pediatrics in review/American Academy of Pediatrics 2002; 23: 264. http://dx.doi.org/10.1542/pir.23-8-264

[14] Horwitz SM, Kelleher KJ, Stein RE, et al. Barriers to the identification and management of psychosocial issues in children and maternal depression. Pediatrics 2007; 119(1): e208-218.

[15] King TM, Tandon SD, Macias MM, et al. Implementing developmental screening and referrals: lessons learned from a national project. Pediatrics 2010; 125(2): 350-360. http://dx.doi.org/10.1542/peds.2009-0388

[16] Ellingson KD, Briggs-Gowan MJ, Carter AS, et al. Parent identification of early emerging child behavior problems: Predictors of sharing parental concern with health providers. Arch Pedatr Adolesc Med 2004; 158: 766-772. http://dx.doi.org/10.1001/archpedi.158.8.766

[17] Hemmeter ML, Santos R, Ostrosky M. A national survey of higher education programs: Preparing early childhood educators to address social emotional development and challenging behavior. Journal of Early Intervention 2008; 30: 321-340.

http://dx.doi.org/10.1177/1053815108320900

[18] Yates T, Ostrosky MM, Cheatham GA, Fettig A, Shaffer L, Santos RM. Research synthesis on screening and assessing social-emotional competence. The Center on Social and Emotional Foundations for Early Learning. Retrieved from vanderbilt.edu/csefel 2008.

[19] Barton EE, Banerjee R. Culturally responsive behavioral supports for children with challenging behaviors and their families. Young Exceptional Children, Monograph \#15: Addressing Young Children's Challenging Behaviors. Los Angeles, CA: Division for Early Childhood 2013.

[20] Shonkoff JP, Phillips DA. From neurons to neighborhoods: The science of early childhood development. National Academies Press 2000.

[21] Godoy L, Carter AS. Identifying and addressing menta health risks and problems in primary care pediatric settings: A model to promote developmental and cultural competence. American Journal of Orthopsychiatry 2013; 83: 73-88. http://dx.doi.org/10.1111/ajop.12005

[22] American Academy of Pediatrics, Task Force on Mental Health. Strategies for system change in children's mental health: A chapter action kit. Elk Grove Gillage IL: American Academy of Pedatrics 2010; Available from www.aap.org/ mentalhealth/mh2ch.html

[23] Squires J, Bricker D, Twombly L. Ages and Stages Questionnaires: Social-Emotional (ASQ:SE): A parentcompleted, child-monitoring system for social-emotional behaviors. Baltimore, MC: Paul H. Brookes Publishing 2002.

[24] Briggs-Gowan M, Carter A. Brief Infant-Toddler Social Emotional Assessment (BITSEA) Manual, version 1.0. New Haven: The Connecticut Early Development Project 2001.

[25] Neisworth JT, Bagnato SJ, Salvia J, Hunt FM. TABS manual for the Temperament and Atypical Behavior Scale. Baltimore, MD: Paul H. Brookes Publishing 1999.

[26] Gokiert RJ, Georgis T, Tremblay M, Krishnan V, Vandenberghe C, Lee C. Evaluating the adequacy of social emotional measures in early childhood. Journal of Psychoeducational Assessment 2014; 32: 441-454. http://dx.doi.org/10.1177/0734282913516718

[27] Bricker D, Schoen Davis M, Squires J. Mental health screening in young children. Infants and Young Children 2004; 25: 62-73.

http://dx.doi.org/10.1097/00001163-200404000-00005

[28] Baggett KM, Warlen L, Hamilton JL, Roberts JL, Staker M. Screening infant mental health indicators: An Early Head Start Initiative. Infants and Young Children 2007; 20: 300310.

http://dx.doi.org/10.1097/01.IYC.0000290353.39793.ba

[29] Division for Early Childhood. DEC Recommended Practices. 2014; Available from: http://www.dec-sped.org/recommendedpractices

[30] Henderson J, Strain P. Screening for social emotional concerns: Considerations in the selection of instruments. Tampa, Florida: University of South Florida, Technical Assistance Center on Social Emotional Intervention for Young Children 2009.

[31] Meyers JC. Developing the work force for an infant and early childhood mental health system of care. In Perry DF, Kaufmann, RK, Knitzer, J, editors. Social and emotional health in early childhood: Building bridges between services and systems. Baltimore, MD: Brookes Publishing 2007.

[32] Kataoka S, Zhang L, Wells K. Unmet need for mental health care among US children: Variation by ethnicity and insurance status. American Journal of Psychiatry 2002; 159: 15-48. http://dx.doi.org/10.1176/appi.ajp.159.9.1548

[33] Burns BJ, Costello EJ, Angold A, et al. Children's mental health service use across service sectors. Health Affairs 1995; 14: 147-159.

http://dx.doi.org/10.1377/hlthaff.14.3.147

[34] Pretti-Frontczak K, Bricker D. An activity-based approach to early intervention. Baltimore, MD: Paul $\mathrm{H}$. Brookes Publishing 2004. 
[35] Squires J, Bricker D, Waddell M, Funk K, Clifford J, Hoselton R. Social-emotional Assessment/Evaluation Measure, Research Edition. Baltimore, MD: Paul H. Brookes Publishing 2014.

[36] NAEYC and NAECS/SDE. Early childhood curriculum, assessment, and program evaluation: building an effective, accountable system in programs for children birth through age 8. 2003; Available from: https://www.naeyc.org/ positionstatements

[37] Division for Early Childhood. Identification of and intervention with challenging behavior. 2009; Available from: http://www.dec-sped.org/papers

[38] National Research Council. Early childhood assessment: Why, what, and how. Committee on Developmental Outcomes and Assessment of Young Children, Snow, CE, Van Hemel, SB, editors, Board on Children, Youth, and Families, Board on Testing and Assessment, Division of Behavioral and Social Sciences Education. Washington, DC: National Academies Press 2008

[39] Neisworth JT, Bagnato SJ. DEC recommended practices: Assessment. In Sandall S, Hemmeter S, Smith ML, McLean, $\mathrm{ME}$, Eds., DEC recommended practices: A comprehensive guide for practical application in early intervention/early childhood special education. Longmont, CO: Sopris West 2005.

[40] Westby CE. Ethnographic interviewing: Asking the right questions to the right people in the right ways. Journal of Children with Communication Disorders 1990; 13: 101-111. http://dx.doi.org/10.1177/152574019001300111

[41] McWilliam RA. Routines-based interview report form Vanderbilt Center for Child Development 2006; Available from http://www.siskin.org/downloads/RBI_Report_Form.pdf

[42] Banerjee R, Guiberson M. Evaluating young children from culturally and linguistically diverse backgrounds for special education services. Young Exceptional Children 2012; 15: 33-45. http://dx.doi.org/10.1177/1096250611435368

[43] Frankland C, Edmonson H, Turnbull AP. Positive behavioral support: Family, school, and community partnerships. Beyond Behavior 2001; Fall: 7-9.

[44] Perez-Mendez C, Moore S. Beyond words: Effective use of translators, interpreters, and cultural mediators 2005.

[45] Ohtake $\mathrm{Y}$, Santos RM, Fowler SA. It's a three way conversation: Families, service providers and interpreters working together. Young Exceptional Children Monograph Series No. 6 on Interdisciplinary Teaming 2005.

DOI: http://dx.doi.org/10.6000/2292-2598.2015.03.04.5

(C) 2015 Steed and Banerjee; Licensee Lifescience Global.

This is an open access article licensed under the terms of the Creative Commons Attribution Non-Commercial License (http://creativecommons.org/licenses/by-nc/3.0/) which permits unrestricted, non-commercial use, distribution and reproduction in any medium, provided the work is properly cited. 